\title{
Consumo de álcool comórbido a transtornos alimentares: uma revisão da literatura
}

\section{Alcohol consumption and eating disorders co-morbidities: a review of the literature}

\author{
Júlio de Carvalho Ponce ${ }^{1}$, Camila Magalhães Silveira², \\ Arthur Guerra de Andrade ${ }^{2,3}$, Lúcio Garcia de Oliveira²
}

\begin{abstract}
Ponce JC, Silveira CM, Andrade AG, Oliveira LG. Consumo de álcool comórbido a transtornos alimentares: uma revisão da literatura. Saúde, Ética \& Justiça. 2011;16(1):30-8.

RESUMO: Introdução: Os transtornos do uso de álcool e os transtornos alimentares podem ser condições comórbidas. Pela escassez de pesquisas brasileiras a respeito dessa associação, uma revisão da literatura foi necessária para melhor compreendê-la. Métodos: Dezenove artigos científicos sobre a relação entre o uso de álcool, seus transtornos e a incidência de transtornos alimentares foram revisados, aprofundando-se a análise em 15 deles. Resultados: Os transtornos alimentares são situações comórbidas ao uso de álcool, de tal forma que a freqüência de seu uso, assim como a prevalência de abuso e dependência, é alta entre pacientes com transtornos alimentares, relação cuja gravidade parece estar aumentada entre os sujeitos diagnosticados com bulimia nervosa. Essa relação também tem sido encontrada entre sujeitos saudáveis que tenham forte restrição sobre a dieta alimentar, um dos sintomas dos transtornos alimentares. Discussão e Conclusões: Como a situação de uma doença adicional pode mudar a sintomatologia, interferir no diagnóstico, no tratamento e no prognóstico de condições psiquiátricas comórbidas, é muito importante que, desde a primeira avaliação de pacientes com suspeita de transtornos alimentares, o uso de álcool seja avaliado, assim como o uso de outras substâncias psicoativas. Detectá-los precocemente pode aumentar a adesão do paciente a um possível tratamento e seu sucesso, assim como a um bom prognóstico.
\end{abstract}

DESCRITORES: Etanol; Transtornos relacionados ao uso de álcool; Alcoolismo; Transtornos da alimentação; Anorexia; Bulimia; Transtorno da compulsão alimentar.

\footnotetext{
1. Departamento de Medicina Legal, Ética Médica e Medicina Social e do Trabalho da Faculdade de Medicina da Universidade de São Paulo - FMUSP.

2. Departamento de Psiquiatria, Faculdade de Medicina da Universidade de São Paulo - FMUSP.

3. Departamento de Psiquiatria da Faculdade de Medicina do ABC - FMABC.

Endereço para correspondência: Lúcio Garcia de Oliveira. Instituto de Psiquiatria. R. Dr. Ovídio Pires de Campos, 785, GREA, 05403-903, São Paulo, SP, Brazil. e-mail: lucgoliver@gmail.com
} 
Ponce JC, et al. Consumo de álcool comórbido a transtornos alimentares: uma revisão da literatura.

\section{INTRODUÇÃO}

$\mathrm{M}$ undialmente, o uso de álcool é responsável por altas taxas de morbidade e mortalidade ${ }^{1}$, destacando-se como um dos fatores de risco mais importantes para a Carga Global de Doenças (Global Burden of Disease - GBD), especialmente na América Latina e Caribe, onde 10\% das mortes e incapacitações têm sido atribuídas ao seu uso ${ }^{2}$.

No Brasil, os estudos epidemiológicos mais abrangentes sobre o uso de álcool, na população geral, foram desenvolvidos pelo Centro Brasileiro de Informações sobre Drogas Psicotrópicas (CEBRID). O CEBRID realizou dois levantamentos domiciliares, nos anos de 2001 e 2005, sobre o uso de substâncias psicotrópicas nas 108 cidades brasileiras com mais de 200 mil habitantes, colhendo amostras significativas de todas as regiões brasileiras, abrangendo todo o território nacional ${ }^{3,4}$. Em ambos os levantamentos, o álcool foi a droga mais prevalentemente consumida, especialmente por homens, tendo sido identificado um aumento do seu uso na vida, de $68,7 \%$ em $2001^{3}$ para $74,6 \%$ em $2005^{4}$, para todas as faixas etárias.

Além da população geral, o uso de álcool também tem sido acompanhado entre estudantes de ensino fundamental, médio e universitário ${ }^{5,6,7,8}$ e aumentos desse uso também têm sido identificados, especialmente para o último segmento social.

Ainda mais preocupante é observar que a porcentagem estimada de dependentes de álcool também tem aumentado, de 11,2\% (em 2001) a $12,3 \%$ (em 2005) ${ }^{3,4}$. Associado a esse aumento de uso e porcentagem de dependência, estão os desdobramentos individuais ao bebedor e à sociedade como um todo. Assim, em levantamento nacional sobre os padrões de consumo de álcool pela população brasileira, Laranjeira et al. ${ }^{9}$ identificaram que $45 \%$ dos brasileiros que declararam ter bebido pelo menos uma vez nos últimos 12 meses, tiveram algum problema relacionado ao uso de álcool. Os problemas físicos foram os mais prevalentes (38\%), seguidos por problemas familiares (18\%), sociais $(17 \%)$, de trabalho (8\%) e legais $(2 \%)$, dos quais $17 \%$ envolveram algum tipo de violência.

Ainda mais preocupante é a constatação de que muitos transtornos psiquiátricos estão relacionados ao uso de álcool e seus transtornos (abusoedependência) ${ }^{10,11,12}$ eque, nessascondições, mesmo o uso de pequenas doses pode desencadear conseqüências mais sérias que as observadas entre pacientes sem co-morbidades ${ }^{13,14}$. Assim, pacientes com co-morbidades, principalmente transtornos psiquiátricos graves, apresentam maiores índices de agressividade, suicídio, recaídas, detenção por atos ilícitos, maiores gastos com tratamentos e reinternações, maior freqüência do uso de serviços médicos, além de apresentarem piora da evolução social e, finalmente, causarem impacto negativo no orçamento familiar e nos acompanhamentos de saúde ${ }^{15}$.

Como a prevalência de co-morbidades psiquiátricas é maior entre as mulheres ${ }^{16,17} \mathrm{e}$ problemas relacionados ao uso de álcool têm correlação com condições relacionadas ao afeto feminino $^{12}$, desperta a curiosidade a relação do uso de álcool com transtornos psiquiátricos especialmente prevalentes entre as mulheres, no caso, os transtornos alimentares.

Conforme o Manual Diagnóstico e Estatístico dos Transtornos Mentais (DSM-IV) ${ }^{18}$, os transtornos alimentares são severas perturbações do comportamento alimentar, identificados especialmente entre as mulheres. Cerca de 0,5\% a $3,0 \%$ da população feminina geral apresenta transtornos alimentares, mas uma porcentagem consideravelmente maior de mulheres apresenta comportamentos alimentares problemáticos e subclínicos $^{19}$.

A Anorexia Nervosa (AN), Bulimia Nervosa (BN), Transtornos Alimentares Sem Outra Especificação (TASOE) e o Transtorno da Compulsão Alimentar Periódica (TCAP) são os principais tipos de transtornos alimentares, porém, os dois últimos não podem ser definidos pelos mesmos critérios diagnósticos dos dois primeiros ${ }^{20}$.

A AN caracteriza-se pela distorção na percepção do próprio corpo, com medo excessivo de engordar (mesmo abaixo do peso; IMC < 17,5 $\mathrm{kg} / \mathrm{m} 2$ ou $<85 \%$ do peso esperado), perda de peso auto-induzida pela privação de alimentos, negação da gravidade de perder peso e, finalmente, distúrbios hipofisários que podem causar amenorréia na mulher e, nos homens, perda de interesse e redução da potência sexual ${ }^{18,21}$. A AN pode ser subdividida em restritiva (quando há apenas a presença de dieta e exercícios para indução da perda de peso) e purgativa (quando há presença de episódios compulsivos e/ou purgativos, como uso de laxantes, indução de hemese, entre outros) ${ }^{22}$. Segundo Claudino e Borges $^{20}$, a BN envolve episódios de perda de controle e ingestão excessiva de alimentos de forma compulsiva e periódica (duas vezes/ semana; no mínimo por três meses consecutivos) e uso de métodos compensatórios para atingir o peso abaixo do recomendado. Subdivide-se em BN purgativa (com indução de vômitos; uso de laxantes, de anorexígenos, hormônios tireoidianos, hormônios diuréticos, entre outros métodos) e BN 
Ponce JC et al. Consumo de álcool comórbido a transtornos alimentares: uma revisão da literatura.

não-purgativa, que emprega jejum e excesso de atividades físicas como métodos compensatórios. Os TASOE preenchem incompletamente critérios para outros transtornos alimentares, geralmente no que se refere à perda de peso e freqüência ou periodicidade do comportamento. Dependendo do transtorno alimentar a que mais se assemelhe, os TASOE podem ser denominados também por ANatípica ou BN-atípica. Por último, o TCAP é definido por uma compulsão alimentar periódica, ou seja, pela ingestão exagerada de alimentos, com perda de controle e angústia associada, não sendo relatada a existência de métodos compensatórios como nas modalidades purgativas da anorexia e bulimia ${ }^{20}$.

A etiopatogenia dos transtornos alimentares é desconhecida, porém, acredita-se em um modelo causal multifatorial, com participação decomponentes biológicos, genéticos, psicológicos, socioculturais e familiares ${ }^{22,23,24,25}$, brevemente citados abaixo:

(a) Fatores genéticos: ainda há controvérsias sobre a magnitude da hereditariedade para 0 desenvolvimento dos transtornos alimentares, mas muitos estudos, entre eles pesquisas conduzidas com gêmeos monozigóticos e dizigóticos apontam a genética como um contribuinte possível à etiologia dos transtornos alimentares; (b) Fatores biológicos: alterações nos níveis normais dos neurotransmissores moduladores da fome e da saciedade (como a noradrenalina, serotonina, colecistoquinina e neuropeptídeos) têm sido vistos como predisponentes ao aparecimento de transtornos alimentares, havendo dúvidas se seriam a origem desses transtornos ou se secundárias de seu quadro clínico; (c) Fatores socioculturais: a obsessão de ter um corpo magro e perfeito é diariamente reforçada pela sociedade ocidental, especialmente pela mídia; (d) Fatores familiares: dificuldades de comunicação, vínculo pobre com os pais, interações impulsivas, divergentes, conflitantes e críticas sobre a aparência dos filhos são fatores de risco à indução e manutenção dos transtornos alimentares; (e) Fatores psicológicos: baixa auto-estima, rigidez de comportamento, distorções cognitivas, controle constante e completo sobre a própria vida e falta de auto-confiança são fatores que podem anteceder o desenvolvimento do quadro clínico característico dos transtornos psiquiátricos.

Os transtornos alimentares são mais prevalentes entre as mulheres $26,27,28,29$. Um estudo realizado no Reino Unido apontou que as mulheres têm um risco 12 vezes maior de desenvolver AN que homens, razão que aumenta para 18 quando considerada a incidência de BN. Independente do gênero e do subtipo de transtorno, a incidência é maior entre os mais jovens ${ }^{26}$. Em função da diferença de incidência de transtornos alimentares entre os gêneros, é comum que algumas pesquisas destinadas a caracterizá-los, façam-no apenas com as mulheres ${ }^{30,31,32}$.

$\mathrm{Na}$ população brasileira, pesquisas têm indicado uma prevalência de $1,1 \%$ de $B N$ entre estudantes do ensino fundamental e médio ${ }^{33}$ e 3,4\% entre universitários ${ }^{34}$. Embora não tenham sido diagnosticados como tal, 13,3\% das estudantes de ensino fundamental e médio apresentaram sintomas de $\mathrm{AN}$ e os mesmos sintomas foram detectados entre $15,6 \%$ de outros adolescentes ${ }^{35}$.

Finalmente, a co-morbidade dos transtornos alimentares e transtornos do uso de álcool tem sido pouco relatada e raros são os estudos brasileiros a respeito. As constatações de que o organismo feminino, para uma mesma quantidade ingerida de álcool, é mais vulnerável aos efeitos de etanol que o organismo masculino ${ }^{36,37}$ e a identificação de uma convergência atual, entre os gêneros, quanto à prevalência do uso de álcool, padrão de uso do tipo "binge drinking" (consumo de 4 ou mais doses de bebidas alcoólicas em uma única ocasião), abuso e dependência ${ }^{38,39,40,41}$ têm sido preocupante. Assim, dado o aumento do uso de álcool entre as mulheres, a maior sensibilidade do organismo feminino aos efeitos dessa substância e considerando-se que os transtornos alimentares são comuns entre as mulheres, a presente revisão teve o propósito de levantar a relação entre ambos os transtornos, dividindo-os conforme seus sub-tipos e destacando sua prevalência, assim como os problemas e/ou dificuldades associados.

\section{MÉTODO}

O presente trabalho consiste em uma revisão da literatura sobre a associação entre transtornos alimentares e o uso, abuso e dependência de álcool, a fim de levantar dados recentes sobre o assunto e proporcionar aos leitores um panorama do tema, sem descartar os eventuais conflitos na literatura. Para isso, artigos científicos publicados entre os anos de 2002 e 2009 foram pesquisados nas bases de dados Medline/PubMed e SciELO. Os descritores utilizados para a pesquisa foram selecionados de acordo com o Medical Subject Headings 2009 (MeSH 2009, http://www.nlm.nih.gov/mesh): eating disorders (transtornos alimentares) OU anorexia OU bulimia OU binge-eating disorder (transtorno da compulsão alimentar periódica) E alcoholrelated disorders (transtornos relacionados ao uso de álcool) OU alcoholism (alcoolismo) OU alcoholinduced disorders, nervous system (transtornos induzidos pelo uso de álcool, sistema nervoso). 
Apesar dos descritores alcohol abuse (abuso de álcool), alcohol dependence (dependência de álcool) e alcohol use disorders (transtornos relacionados ao uso de álcool) não constarem no MeSH 2009, eles foram também utilizados nas buscas por serem amplamente escolhidos como palavraschave nos artigos. Aplicaram-se diferentes filtros simultaneamente, com os resultados das pesquisas contendo estudos publicados nos idiomas inglês, português e espanhol, e realizados com humanos do gênero feminino - uma vez que os transtornos alimentares são mais prevalentes neste gênero e, portanto, existe um maior número de publicações relacionadas. Estudos divulgados antes do período delimitado foram incluídos quando se tratava de artigos pioneiros ou de revisões importantes sobre transtornos alimentares ou transtornos relacionados ao uso de álcool. Também foram revisadas algumas das referências bibliográficas encontradas nos principais artigos científicos pesquisados, assim como livros especializados.

\section{RESULTADOS E DISCUSSÃO}

Muitos transtornos psiquiátricos têm sido relacionados ao uso, abuso e dependência de álcool, especialmente entre as mulhere ${ }^{11}$, por vezes precedendo o próprio consumo de álcool ${ }^{42,43}$, mas cuja relação temporal ainda permanece controversa. O tipo de transtorno psiquiátrico comórbido aos transtornos do uso de álcool parece sofrer influência do gênero, de tal forma que, enquanto os transtornos de internalização (depressão e ansiedade) estão habitualmente associados ao consumo de álcool entre mulheres, os transtornos de externalização (transtornos de personalidade anti-social e dependência de drogas) são mais comuns entre os homens ${ }^{16}$. Para ilustrar, recentemente, relação semelhante foi encontrada em estudo conduzido com estudantes universitárias, em que os problemas decorrentes do consumo excessivo de álcool estavam associados exclusivamente à esfera afetiva dessas estudantes ${ }^{12}$.

Especificamente quanto aos transtornos alimentares, sãocondiçõespossivelmentecomórbidas a outros diagnósticos psiquiátricos como transtornos de humor, ansiedade, síndrome do pânico, episódios de mania e uso de substâncias psicotrópicas ${ }^{29,30}$. Quanto ao uso de substâncias, mulheres com risco de desenvolver um transtorno alimentar, identificado, por exemplo, como um escore igual ou superior a 20 pontos na escala EAT-26 (Eating Attitude Test - total de 78 pontos possíveis), têm uma chance três vezes maior de desenvolverem dependência a alguma substância quando comparadas a mulheres sem esse risco ${ }^{29}$. Diretamente a respeito do uso de álcool, na Europa, especialmente na Espanha, na Áustria e na Eslovênia, a prevalência do uso de álcool na vida é maior entre pacientes com algum transtorno alimentar em relação a sujeitos saudáveis (34,1\% vs. $26,9 \%$; $p=0,002)^{28}$, sugerindo a existência de uma relação entre esses transtornos.

Em uma metanálise sobre a ocorrência simultânea de transtornos alimentares e transtornos relacionados ao uso de álcool, conduzida sobre os resultados de 41 pesquisas, apenas 4 dos estudos analisados não haviam identificado esse tipo de associação ${ }^{44}$. Esse estudo apontou que pacientes com transtornos alimentares apresentaram maior chance de consumir álcool com regularidade e de ter sensação de perda de controle sobre a quantidade e a freqüência de uso, especialmente as pacientes diagnosticadas com bulimia nervosa (BN) $(77,1 \%$ dos casos de BN; $64,6 \%$ de AN purgativa; $53,5 \%$ de TASOE; $42,9 \%$ de AN restritiva) ${ }^{28}$.

Em relação ao abuso e à dependência de álcool, tais condições são identificadas entre 16\% das pacientes com transtornos alimentare ${ }^{30}$. Quanto aos seus subtipos, novamente, identifica-se que a bulimia nervosa (BN) tem maior associação com os transtornos relacionados ao uso de álcool (provavelmente pela relação que ambas as condições têm com comportamentos compulsivos), seguida de outros transtornos purgativos, TASOE e TCAP ${ }^{10}$. Em termos de prevalência, Blinder et $a .^{30}$ sugeriram que o abuso/dependência de álcool esteja presente em $16 \%$ dos casos de BN, $14 \%$ de AN purgativa, $14 \%$ de TASOE e $3 \%$ de AN restritiva, mas realmente parece haver uma flutuação dessas prevalências, de tal forma que Franko et al. ${ }^{45}$ apontaram os seguintes valores: (a) BN: de 2,9 a 48,6\%; (b) AN restritiva: 2,0 a $6,0 \%$ e AN purgativa: 12,0 a $27,0 \%$.

Essa associação com a BN já havia sido estabelecida no trabalho pioneiro de Dansky et al. ${ }^{31}$. Ao analisar uma amostra nacionalmente representativa de 3.006 mulheres dos Estados Unidos, classificando os problemas relacionados ao álcool em Abuso de Álcool (AA: identificado como ter problemas no trabalho, com amigos, com a família ou com a polícia devido ao uso de álcool) ou Dependência de Álcool (DA), verificou-se que a BN esteve mais associada a esses problemas do que outros transtornos alimentares. Ainda nessa população, quase um terço (31\%) das mulheres com histórico de BN também apresentaram histórico de AA, e cerca de um oitavo (13\%) apresentou quadro de DA. Outro achado importante foi que mulheres com quadro de BN e AA tinham maior prevalência de uso de laxantes e indução de vômitos, além de histórico de abuso sexual e maior prevalência do uso de tabaco ${ }^{31}$. 
Ponce JC et al. Consumo de álcool comórbido a transtornos alimentares: uma revisão da literatura.

Outro estudo realizado em 2007 sugeriu um maiorconsumo deálcool entre sujeitos com TCAP (em relação aos com TASOE e aos sujeitos saudáveis), mas não em relação a sujeitos diagnosticados com BN $(p<0,01)$. No entanto, ao analisar os motivos que levavam as pessoas a beberem, houve uma maior prevalência no prejuízo do coping (enfrentamento de situações desconfortáveis) entre sujeitos com BN e TCAP do que entre sujeitos com TASOE e sujeitos saudáveis, indicando que os primeiros grupos citados podem desenvolver uma relação patológica com o uso de álcool. Os autores ainda ressaltaram que o baixo número de casos de BN pode ter impedido que diferenças estatisticamente significativas fossem encontradas para as medidas adotadas para mensurar o consumo de álcool ${ }^{46}$.

Porém, em uma pesquisa conduzida com 217 mulheres diagnosticadas com BN, foi descrito um subtipo, denominado por multi-impulsivo (com uma série de comportamentos associados como uso e abuso de substâncias, tentativas de suicídio, comportamentos anti-sociais, ferimentos autoprovocados, entre outros), caracterizado por uma maior freqüência de uso de álcool que o restante das pacientes ${ }^{47}$, sugerindo que a situação pode ser mais complicada para esse subtipo de transtorno.

Para Franko et al. ${ }^{45}$, a relação entre os transtornos alimentares e os transtornos relacionados ao uso de álcool é de mão dupla, parecendo haver maior influência dos transtornos alimentares sobre os transtornos do uso de álcool que o inverso. Porém, em um estudo canadense realizado com 36.984 sujeitos (20.211 mulheres e 16.773 homens), observou-se uma associação estatisticamente significativa entre o risco de desenvolvimento de transtornos alimentares e o acontecimento de uma conseqüência negativa do uso de álcool nos 12 meses anteriores à entrevista, ou seja, envolver-se em situações nas quais o álcool teve influência negativa na rotina, nas funções acadêmicas/profissionais e/ou relações pessoais, facilitou o aparecimento subseqüente de transtornos alimentares ${ }^{27}$.

Contrário ao que se poderia esperar, a relação entre os transtornos alimentares e os transtornos relacionados ao uso de álcool está longe de ser exclusividade das mulheres mais jovens. A esse respeito, uma pesquisa canadense estudou o risco de desenvolver um transtorno alimentar e dependência de álcool entre mulheres de diferentes faixas etárias (15-24; 25-44; superior a 44 anos), identificando essa associação para todas as faixas etárias investigadas, especialmente entre as mulheres com mais de 25 anos $^{32}$. Outro estudo canadense realizado com mais de 9 mil mulheres com idade superior a 50 anos observou que a chance de ser dependente de álcool, entre mulheres de 50 a 64 anos e com sintomas de transtorno alimentar, é seis vezes maior que para as mulheres sem esta sintomatologia ${ }^{48}$.

Outra constatação relevante é que a presença de comportamentos (ou sintomas) isolados de transtornos alimentares pode ter uma relação positiva com o consumo nocivo de álcool, como já observado para o sintoma de preocupação excessiva com o peso, insatisfação com o corpo e comportamentos compulsivos ou compensatórios com a alimentação ${ }^{12}$. Em pesquisa realizada com 255 universitárias norte-americanas, aquelas que desejavam perder peso não tinham um maior risco de desenvolver padrões nocivos de consumo de álcool, mas apresentavam maior número de relatos de conseqüências nocivas advindas desse uso, em especial machucar a si própria, fazer algo de que se arrependera e ter relações sexuais forçadas ${ }^{19}$. Para reforçar essa constatação, um estudo realizado com universitárias de primeiro ano, categorizadas conforme sua prática de dietas (não praticante; praticantes casuais; praticantes intensas e praticantes em risco de desenvolver BN), apontou que praticantes intensas e de risco apresentavam maior prevalência de uso de álcool, de já terem se embriagado na vida e de terem sofrido problemas relacionados ao álcool nos seis meses anteriores à pesquisa. Em relação às conseqüências que já houvessem sofrido, as mais frequentemente relatadas entre as praticantes de risco foram: beber mais do que o planejado; ter blackouts ou "apagões" ao beber; atividades sexuais não desejadas; ter a necessidade de fazer uso de maiores quantidades de álcool para ter o mesmo efeito que anteriormente tinha (efeito de tolerância); ter recebido "reclamações de terceiros" e "já ter dirigido embriagada" 4 .

Quanto ao binge eating ou compulsão alimentar, este pode ser definido como o padrão de comer sem limites, com fissuras e com gratificação imediata, é um comportamento que também causa dependência e gera danos a longo-prazo. A associação entre o padrão "binge" de alimentação e transtornos relacionados ao uso de álcool já foi identificada entre universitárias, em que se observou que estudantes que se alimentavam nesse padrão bebiam de forma pesada (heavy episodic drinking: 5 ou mais doses em uma única ocasião) e também apresentavam comportamentos anti-sociais e impulsividade ${ }^{50}$. Finalmente, em estudo com 517 mulheres, identificou-se que esse comportamento alimentar compulsivo, quando grave, estava associado ao uso excessivo de álcool quando comparado a outras expressões inadequadas de comportamento alimentar ${ }^{51}$. 
Ponce JC, et al. Consumo de álcool comórbido a transtornos alimentares: uma revisão da literatura.

Embora a associação entre transtornos alimentares e transtornos relacionados ao uso de álcool seja mais prevalente entre as mulheres, também tem sido identificada entre os homens ${ }^{27}$. Além disso, entre mulheres, o desenvolvimento de transtornos alimentares é facilitado pela existência de antecedentes familiares de alcoolismo ${ }^{12}$, fortalecendo as evidências sobre essa possível associação.

Com finalidade ilustrativa, a Tabela 1 contém o resumo dos artigos científicos detalhados nessa revisão.

Tabela 1. Resumo dos artigos científicos

\begin{tabular}{|c|c|c|c|c|c|}
\hline Referência & País & População & Prevalência de TA & $\begin{array}{l}\text { Prevalência de uso, abuso e } \\
\text { dependência de álcool e outras } \\
\text { substâncias }\end{array}$ & Associação \\
\hline $\begin{array}{l}\text { Dansky et } \\
\text { al. }{ }^{31}\end{array}$ & $\begin{array}{l}\text { Estados } \\
\text { Unidos }\end{array}$ & $\begin{array}{l}3.006 \\
\text { mulheres }\end{array}$ & $\begin{array}{l}2,4 \% \text { - mulheres com } \\
\text { Bulimia Nervosa }\end{array}$ & $\begin{array}{l}22 \% \text { - mulheres com abuso de } \\
\text { álcool } \\
6,3 \% \text { - mulheres com dependência } \\
\text { de álcool }\end{array}$ & $\begin{array}{l}\text { Dependência de álcool e Bulimia } \\
\text { Nervosa }(31 \%)^{*} \\
\text { [Controles sem bulimia- } 18,8 \% \text { de } \\
\text { dependentes de álcool] }\end{array}$ \\
\hline $\begin{array}{c}\text { Piran, } \\
\text { Robinson }\end{array}$ & Canadá & 517 mulheres & $\begin{array}{l}11 \% \text { - binge severo } \\
\text { (últimos } 12 \text { meses) } \\
29 \% \text { - binge (últimos } 12 \\
\text { meses) } \\
5 \% \text {-dieta severa } \\
\text { (últimos } 12 \text { meses) } \\
39 \% \text { - dieta (últimos } 12 \\
\text { meses) }\end{array}$ & $\begin{array}{l}75 \% \text { - uso leve a moderado } \\
21 \% \text { - uso abusivo }\end{array}$ & $\begin{array}{l}\text { Abuso de álcool e binge severo } \\
(25 \%)^{\star} ; \\
\text { Abuso de álcool, binge severo e dieta } \\
\text { sem comportamentos purgativos } \\
(33 \%)^{\star} ; \\
\text { Abuso de álcool, binge, dieta e } \\
\text { comportamentos purgativos }(26 \%)^{\star} ; \\
\text { Abuso de álcool, binge severo, dieta e } \\
\text { comportamentos purgativos }(30 \%)^{*} ; \\
\text { Abuso de álcool, binge severo, } \\
\text { dieta severa e comportamentos } \\
\text { purgativos }(34 \%)^{\star} \text {. } \\
\text { [controles: } 15 \% \text { de abuso de álcool] }\end{array}$ \\
\hline $\begin{array}{l}\text { Gadalla, } \\
\text { Piran }^{27}\end{array}$ & Canadá & $\begin{array}{l}16.773 \\
\text { homens } \\
20.211 \\
\text { mulheres }\end{array}$ & $\begin{array}{l}2,8 \% \text { - mulheres com } \\
\text { risco de desenvolver TA } \\
\text { (EAT-26>20) (últimos } \\
12 \text { meses) } \\
0,5 \% \text { - homens com } \\
\text { risco de desenvolver TA } \\
\text { (EAT-26>20) (últimos } \\
12 \text { meses) }\end{array}$ & $\begin{array}{l}\text { 3,9\% - homens com dependência } \\
\text { de álcool } \\
1,3 \% \text { - mulheres com dependência } \\
\text { de álcool } \\
2,2 \% \text { - mulheres com interferência } \\
\text { do álcool } \\
\text { 0,7\% - homens com interferência } \\
\text { do álcool }\end{array}$ & $\begin{array}{l}\text { Dependência do álcool e EAT-26>20 } \\
\text { para homens*; } \\
\text { Dependência do álcool e EAT-26>20 } \\
\text { para mulheres } \\
\text { Interferência do álcool e EAT-26>20 } \\
\text { para homens }{ }^{* * * * ;} \\
\text { Interferência do álcool e EAT- } \\
26>20^{* * * *} \text {. }\end{array}$ \\
\hline Gadalla $^{48}$ & Canadá & $\begin{array}{l}9.014 \\
\text { mulheres } \\
\text { acima de } 50 \\
\text { anos }\end{array}$ & $\begin{array}{l}2,6 \% \text { - mulheres de } 50 \\
\text { a } 64 \text { anos com risco de } \\
\text { desenvolver TA (EAT- } \\
26>20) \\
1,8 \% \text { - mulheres acima } \\
\text { de } 64 \text { anos com risco } \\
\text { de desenvolver TA } \\
(\text { EAT-26>20) }\end{array}$ & $\begin{array}{l}0,3 \% \text { - mulheres entre } 50 \text { a } 64 \text { anos } \\
\text { com dependência de álcool }\end{array}$ & $\begin{array}{l}\text { Mulheres entre } 50 \text { a } 64 \text { anos com } \\
\text { risco de desenvolver TA (EAT-26>20) } \\
\text { tem OR de } 6.0 \text { em relação a mulheres } \\
\text { com EAT- } 26<20 \text { para dependência de } \\
\text { álcool }^{*} \text {. }\end{array}$ \\
\hline $\begin{array}{l}\text { Gadalla, } \\
\text { Piran }^{22}\end{array}$ & $\begin{array}{l}\text { Meta- } \\
\text { análise }\end{array}$ & $\begin{array}{l}101.081 \\
\text { mulheres }\end{array}$ & - & - & $\begin{array}{l}\text { Transtornos relacionados ao uso } \\
\text { de álcool estão mais fortemente } \\
\text { associados a Bulimia Nervosa, } \\
\text { seguido de comportamentos } \\
\text { purgativos, TASOE e TCAP. }\end{array}$ \\
\hline $\begin{array}{l}\text { Dams- } \\
\text { O'Connor et } \\
\text { al. }^{19}\end{array}$ & $\begin{array}{l}\text { Estados } \\
\text { Unidos }\end{array}$ & $\begin{array}{l}255 \\
\text { universitárias }\end{array}$ & $\begin{array}{l}49,8 \% \text { - universitárias } \\
\text { que estavam tentando } \\
\text { perder peso } \\
50,2 \% \text { - universitárias } \\
\text { que não estavam } \\
\text { tentando perder peso }\end{array}$ & $\begin{array}{l}0,9 \% \text { - feriram outra pessoa devido } \\
\text { ao álcool } \\
34 \% \text { - fizeram algo de que se } \\
\text { arrependeram devido ao álcool }\end{array}$ & $\begin{array}{l}\text { Mulheres que estavam tentando } \\
\text { perder peso se feriram }\left(10,8 \%^{*}\right) \text {, } \\
\text { fizeram algo do qual se arrependeram } \\
\left(47,1 \% \%^{* *}\right) \text { e tiveram relações sexuais } \\
\text { forçadas }\left(17,6 \%^{* * *}\right) \text { devido ao } \\
\text { álcool com maior frequência do que } \\
\text { mulheres que não estavam tentando } \\
\text { perder peso }\end{array}$ \\
\hline
\end{tabular}

continua 
Ponce JC et al. Consumo de álcool comórbido a transtornos alimentares: uma revisão da literatura.

\begin{tabular}{|c|c|c|c|c|c|}
\hline Referência & País & População & Prevalência de TA & $\begin{array}{l}\text { Prevalência de uso, abuso e } \\
\text { dependência de álcool e outras } \\
\text { substâncias }\end{array}$ & Associação \\
\hline Keel et al. ${ }^{52}$ & $\begin{array}{l}\text { Estados } \\
\text { Unidos }\end{array}$ & $\begin{array}{l}246 \text { mulheres } \\
\text { com bulimia } \\
\text { ou anorexia }\end{array}$ & $\begin{array}{l}55,3 \% \text { - mulheres com } \\
\text { anorexia nervosa } \\
44,7 \% \text { - mulheres com } \\
\text { bulimia nervosa }\end{array}$ & $\begin{array}{l}2 \text { das } 11 \text { mulheres que morreram } \\
\text { tiveram uma causa mortis } \\
\text { diretamente relacionada ao álcool }\end{array}$ & $\begin{array}{l}\text { Houve associação do tempo até a } \\
\text { morte com a severidade do uso de } \\
\text { álcool** }^{\star * *}\end{array}$ \\
\hline $\begin{array}{l}\text { Blinder et } \\
\text { al. }^{30}\end{array}$ & $\begin{array}{l}\text { Estados } \\
\text { Unidos }\end{array}$ & $\begin{array}{l}2436 \\
\text { pacientes } \\
\text { com } \\
\text { diagnóstico } \\
\text { de } \\
\text { transtornos } \\
\text { alimentares }\end{array}$ & $\begin{array}{l}21,3 \% \text { pacientes com } \\
\text { ANR } \\
17,9 \% \text { pacientes com } \\
\text { ANP } \\
36,2 \% \text { pacientes com } \\
\text { BN } \\
24,6 \% \text { pacientes com } \\
\text { TASOE } \\
\end{array}$ & $\begin{array}{l}\text { Abuso/dependência de álcool: } \\
\text { Geral }-16 \% \\
\text { BN }-26 \% \\
\text { ANR }-3 \% \\
\text { ANP }-14 \% \\
\text { TASOE - } 14 \%\end{array}$ & $\begin{array}{l}\text { Pacientes com Bulimia Nervosa } \\
\text { tem maior prevalência de Abuso/ } \\
\text { dependência de álcool *** } \\
\text { Pacientes com Bulimia Nervosa tem } 2 \\
\text { vezes mais chance de terem Abuso/ } \\
\text { Dependência de álcool (ajustado por } \\
\text { análise multivariada) }\end{array}$ \\
\hline Krug et al. ${ }^{28}$ & $\begin{array}{l}\text { Reino } \\
\text { Unido, } \\
\text { Espanha, } \\
\text { Austria, } \\
\text { Eslovenia } \\
\text { e Itália }\end{array}$ & $\begin{array}{l}879 \text { pacientes } \\
\text { com TA }\end{array}$ & $\begin{array}{l}172 \text { - pacientes com } \\
\text { ANR } \\
156 \text { - pacientes com } \\
\text { ANP } \\
250 \text { - pacientes com BN } \\
201 \text { - pacientes com } \\
\text { TASOE }\end{array}$ & $\begin{array}{l}\text { Alguma vez na vida bebeu álcool } \\
\text { regularmente: } 34,1 \% \text { TA } \\
\text { Perda de controle sobre a bebida } \\
\text { (na vida): } 57,9 \% \text { TA } \\
\text { Perda de controle sobre a bebida } \\
\text { (atualmente): } 24,4 \% \text { TA [ ANR- } \\
\text { 17,7\%; ANP- } 31,8 \% \text {; BN- } 29,2 \% \text {; } \\
\text { TASOE- } 25,6 \% \text { ] }\end{array}$ & $\begin{array}{l}\text { Uso na vida de álcool foi maior em TA } \\
(\mathrm{OR}=1,40)^{\star * *} \\
\text { Perda de controle sobre uso de álcool } \\
\text { na vida foi maior em TA }(\mathrm{OR}=6,10)^{* *} \\
\text { BN tem maior prevalência de perda de } \\
\text { controle sobre uso de álcool na vida } \\
\text { em comparação com outras TAs } \\
\text { Pacientes com TASOE tem maior } \\
\text { consumo atual de álcool } \\
\text { Perda de controle sobre uso de } \\
\text { álcool atualmente é maior para TA } \\
(\mathrm{OR}=3,04)^{* \star}\end{array}$ \\
\hline $\begin{array}{l}\text { Krahn et } \\
\text { al. }^{49}\end{array}$ & $\begin{array}{l}\text { Estados } \\
\text { Unidos }\end{array}$ & $\begin{array}{l}1384 \\
\text { universitárias }\end{array}$ & $\begin{array}{l}8 \% \text { - mulheres sem } \\
\text { dieta } \\
26 \% \text { - mulheres com } \\
\text { dietas casuais } \\
44 \% \text { - mulheres com } \\
\text { dietas intensas } \\
22 \% \text { - mulheres com } \\
\text { dietas de risco }\end{array}$ & $\begin{array}{l}90 \% \text { - usou álcool pelo menos uma } \\
\text { vez na vida } \\
61 \% \text { - consumiu álcool no mês } \\
\text { anterior } \\
35 \% \text { - bebem pelo menos uma vez } \\
\text { por semana } \\
\text { Das que haviam experimentado } \\
\text { álcool: } \\
26 \% \text { - costumam beber até ficar } \\
\text { embriagadas na maioria das } \\
\text { ocasiões } \\
28 \% \text { - beberam } 5 \text { ou mais doses } \\
\text { em uma só ocasião nas ultimas } \\
\text { duas semanas } \\
41 \% \text { - tiveram pelo menos uma } \\
\text { consequência negativa do consumo } \\
\text { de álcool no mês anterior }\end{array}$ & $\begin{array}{l}\text { RR (em relação às mulheres que não } \\
\text { dietam) } \\
\text { Prevalência de uso de álcool no mês } \\
\text { anterior: } \\
\text { Dieta intensa - } 1,34^{* * *} \text {; Dietas de risco } \\
-1,48^{\star * \star *} \text {. } \\
\text { Prevalência de embriaguez na vida: } \\
\text { Dieta intensa - } 1,70^{\star * *} \text {; Dietas de risco } \\
-2,09^{\star \star \star *} \text {. } \\
\text { Prevalência de uso de } 5 \text { ou mais } \\
\text { doses em uma ocasião (nas ultimas } \\
\text { duas semanas): } \\
\text { Dieta intensa - } 3,26^{\star} \text {. } \\
3 \text { ou mais consequencias negativas do } \\
\text { álcool nos ultimos } 6 \text { meses: } \\
\text { Dieta intensa - } 1,18^{\star} ; \text { Dietas de risco } \\
-1,23^{\star *} \text {. }\end{array}$ \\
\hline Luce et al. ${ }^{46}$ & $\begin{array}{l}\text { Estados } \\
\text { Unidos }\end{array}$ & $\begin{array}{l}383 \\
\text { universitárias }\end{array}$ & $\begin{array}{l}\text { 4,2\% - Bulimia Nervosa } \\
\text { 7,8\% - TCAP } \\
22,2 \% \text { - TASOE } \\
65,8 \% \text { - sem TA }\end{array}$ & $\begin{array}{l}\text { Média de doses no fim de semana: } \\
\text { BN }-6,33 \\
\text { TCAP - } 8,03 \\
\text { TASOE }-5,45 \\
\text { sem TA - } 4,89\end{array}$ & $\begin{array}{l}\text { TCAP consomem mais doses no fim } \\
\text { de semana do que TASOE e sem TA }\end{array}$ \\
\hline $\begin{array}{l}\text { Franko et } \\
\text { al. }^{45}\end{array}$ & $\begin{array}{l}\text { Estados } \\
\text { Unidos }\end{array}$ & $\begin{array}{l}246 \text { mulheres } \\
\text { com bulimia } \\
\text { ou anorexia }\end{array}$ & $\begin{array}{l}136 \text { - mulheres com AN } \\
110 \text { - mulheres com BN }\end{array}$ & $\begin{array}{l}\text { Transtornos relacionados ao uso } \\
\text { de álcool: } \\
17 \% \text { - apresentaram no início do } \\
\text { estudo } \\
27 \% \text { - apresentaram os transtornos } \\
\text { após } 9 \text { anos }\end{array}$ & $\begin{array}{l}\text { Não houve diferença de prevalência } \\
\text { entre os dois grupos }\end{array}$ \\
\hline $\begin{array}{l}\text { Benjamin, } \\
\text { Wulfert }^{50}\end{array}$ & $\begin{array}{l}\text { Estados } \\
\text { Unidos }\end{array}$ & $\begin{array}{l}335 \\
\text { universitárias }\end{array}$ & $\begin{array}{l}\text { Episódio de binge } \\
\text { eating no mês anterior: } \\
76,7 \% \text { - sem episódios } \\
\text { de binge } \\
9,9 \%-1 \text { ou } 2 \text { episódios } \\
6,3 \% \text { - } 3 \text { a } 5 \text { episódios } \\
7,2 \% \text { - mais de } 5 \\
\text { episódios }\end{array}$ & $\begin{array}{l}\text { Consumo de álcool no mês anterior } \\
21,8 \% \text { - não consumiram álcool } \\
20,3 \% \text { - } 1 \text { ou } 2 \text { dias } \\
20,6 \% \text { - } 3 \text { a } 5 \text { dias } \\
17,9 \% \text { - } 6 \text { a } 9 \text { dias } \\
19,7 \% \text { - } 10 \text { ou mais dias } \\
55,8 \% \text { - bebedoras binge (4 ou mais } \\
\text { doses em uma ocasião) } \\
22,4 \% \text { - bebedoras binge frequentes } \\
\text { (3 ou mais episódios de binge) }\end{array}$ & $\begin{array}{l}\text { Houve correlação entre a } \\
\text { frequência de binge eating } \\
\text { e uso de álcool }\end{array}$ \\
\hline
\end{tabular}

${ }^{*} \mathrm{p}<0,05 ; \quad{ }^{* *} \mathrm{p}<0,01 ;{ }^{* * \star} \mathrm{p}<0,005 ;{ }^{* * *} \mathrm{p}<0,0005$; TA- Transtornos Alimentares; BN- Bulimia Nervosa; AN- Anorexia Nervosa; ANR- Anorexia Nervosa Restritiva; ANP- Anorexia Nervosa Purgativa; TASOE- Transtornos Alimentares Sem Outra Especificação; TCAP- Transtorno Compulsivo Alimentar Periódico. 


\section{CONSIDERAÇÕES FINAIS}

Poucos estudos brasileiros têm retratado a prevalência dos transtornos alimentares (seja na população geral ou em sub-populações específicas) e menor tem sido o número de pesquisas que têm investigado a relação entre a incidência de transtornos alimentares e transtornos relacionados ao uso de álcool. Entretanto, pesquisas e levantamentos internacionais já têm apontado que mulheres sob risco de desenvolver transtornos alimentares ou já diagnosticadas como tal, usam álcool em maior quantidade e freqüência, podendo fazê-lo com regularidade e pouco controle, já tendo sido descrita a co-morbidade dos transtornos alimentares com o abuso e dependência de álcool. Embora essa associação tenha sido identificada entre homens, continua sendo mais prevalente entre mulheres, independentemente de sua faixa etária. Essa associação parece ser uma via de mão dupla e, embora uma relação causal já tenha sido sugerida, ainda é controversa. Além disso, não parece ser necessário que todos os sintomas necessários para se ter diagnóstico de transtorno alimentar estejam presentes, bastando apenas um sintoma importante (ex.: dieta severa ou de risco) para que uma associação negativa com o uso de álcool ou com as conseqüências dele advindas seja identificada. Finalmente, quanto ao subtipo de transtorno alimentar, parece consensual que a associação com os transtornos relacionados ao uso de álcool seja mais intensa entre sujeitos diagnosticados com bulimia nervosa. Por fim, ao identificar que o beber pesado, abuso e dependência de álcool sejam fatores relacionados ao mau prognóstico, cronificação dos transtornos, morbidade e mortalidade entre pacientes com transtornos alimentares, essa freqüente associação deve ser vista como um problema de saúde pública. Estudos nacionais capazes de identificar a associação entre transtornos alimentares e transtornos relacionados ao uso de substâncias em nossa população são necessários a fim de que possamos identificar os problemas e o impacto decorrentes desta associação em nossa população a fim de guiar políticas públicas.

Ponce JC, Silveira CM, Andrade AG, Oliveira LG. Alcohol consumption and eating disorders co-morbidities: a review of the literature. Saúde, Ética \& Justiça. 2011;16(1):30-8.

\begin{abstract}
Introduction: Alcohol use disorders and eating disorders can be comorbid conditions. Due to the scarcity of Brazilian studies analyzing this association, a review of the literature was necessary to better understand it. Methods: Nineteen research reports on the relationship between alcohol use, its disorders and the incidence of eating disorders were reviewed, and of those, fifteen were analyzed in details. Results: Eating disorders are generally co morbid to alcohol use, in such a way that its frequency of use, as well as its prevalence of abuse and dependence, is high among patients with eating disorders, a relationship which seems to increase in severity among patients with bulimia nervosa. Such relationship has also been found among healthy subjects with a strong restriction on dietary behavior, one of the symptoms of eating disorders. Discussion and Conclusions: Since the status of an additional disease can change the symptomatology, the diagnosis, the treatment and the prognosis of comorbid psychiatric conditions, it is very important that, from a patients' first evaluation of suspected eating disorders, alcohol used be evaluated, as well as the other drugs use. Detecting them early on can increase the adherence of a patient to a possible treatment and its success, as well as a good prognosis.
\end{abstract}

KEY WORDS: Ethanol; Alcohol-related disorders; Alcoholism; Eating disorders; Anorexia; Bulimia; Binge-eating disorders.

\section{REFERÊNCIAS}

1. Ezzati M, Lopez AD, Rodgers A. Selected major risk factors and global and regional burden of disease. Lancet. 2002;360(9343):1347-60.

2. Lopez AD, Mathers CD, Ezzati M, Jamison DT, Murray CJL. Global and regional burden of disease and risk factors, 2001: systematic analysis of population health data. Lancet. 2006;367(9524):1747-57.

3. Carlini EA, Galduróz JCF, Noto AR, Nappo AS.
I levantamento domiciliar sobre o uso de drogas psicotrópicas no Brasil. São Paulo: Centro Brasileiro de Informações sobre Drogas Psicotrópicas (CEBRID), Secretaria Nacional Antidrogas (SENAD); 2002.

4. CEBRID - Centro Brasileiro de Informações sobre Drogas Psicotrópicas. II Levantamento Domiciliar sobre o uso de drogas psicotrópicas no Brasil: estudo envolvendo as 108 maiores cidades do 
Ponce JC et al. Consumo de álcool comórbido a transtornos alimentares: uma revisão da literatura.

país. São Paulo: Centro Brasileiro de Informações sobre Drogas Psicotrópicas (CEBRID) e Secretaria Nacional Antidrogas (SENAD); 2007.

5. Galduróz JCF, Caetano R. Epidemiology of alcohol use in Brazil. Rev Bras Psiquiatr. 2004;26(Supl I):3-6.

6. Andrade AG, Queiroz S, Villaboim RCM, César CLG, Alves MCGP, Bassit AZ. Uso de álcool e drogas entre alunos de graduação da Universidade de São Paulo. Rev ABP-APAL. 1997;19(2):53-9.

7. Stempliuk VA, Barroso LP, Andrade AG, Nicastri S, Malbergier A. Comparative study of drug use among undergratuate students at the University of São Paulo - São Paulo campus in 1996 and 2001. Rev Bras Psiquiatr. 2005;27(3):185-93.

8. Wagner GA, Andrade, AG. Uso de álcool, tabaco e outras drogas entre estudantes universitários brasileiros. Rev Psiquiatr Clin. 2008;35 (1):48-54.

9. Laranjeira R, Pinsky I, Zaleski M, Caetano R. I Levantamento Nacional sobre os padrões de consumo de álcool na população brasileira. Brasília: Secretaria Nacional Antidrogas; 2007.

10. Holderness CC, Brooks-Gunn J, Warren MP. Comorbidity of eating disorders and substance abuse review of the literature. Int J Eat Disord. 1994;16:134.

11. Cornelius JR, Salloum IM, Mezzich J, Cornelius MD, Fabrega H Jr, Ehler JG, Ulrich RF, Thase ME and Mann JJ. Disproportionate suicidality in patients with comorbid major depression and alcoholism. Am J Psychiatry. 1995;152:358-64

12. Harrell ZAT, Slane JD, Klump KL. Predictors of alcohol problems in college women: the role of depressive symptoms, disordered eating, and family history of alcoholism. Addict Behav. 2009;34:252-7.

13. Drake RE, Osher FC, Wallach MA. Alcohol use and abuse in schizophrenia. A prospective community study. J Nerv Ment Dis. 1989;177(7):408-14.

14. Menezes PR, Johnson S, Thonicroft G, Marshall $J$, Prosser D, Bebbington P, Kuipers E. Drug and alcohol problems among individuals with severe mental illness in south London. $\mathrm{Br} \mathrm{J}$ Psychiatry. 1996; 168(5):612-9.

15. Clark RE, Drake RE. Expenditures of time and money by families of people with several mental illness and substance use disorders. Commun Ment Health J. 1994;30:145-63.

16. Hesselbrock, MN, Meyer, RE, Keener, JJ. Psychopathology in hospitalized alcoholics. Arch Gen Psychiatry. 1985;42:1050-5.

17. Rounsaville BJ, Donlinsky ZS, Babor TF, Meyer RE. Psychopathology as a predictor of treatment outcome in alcoholics. Arch Gen Psychiatry. 1987;44:505-13.
18. American Psychiatric Association. Diagnostic and statistical manual of mental disorders DSM-IV. Washington (DC); 1994.

19. Dams-O'Connor K, Martens MP, Anderson DA. Alcohol-related consequences among women who want to lose weight. Eating Behav. 2006;7:188-95.

20. Claudino AM, Borges MBF. Critérios diagnósticos para os transtornos alimentares: conceitos em evolução. Rev Bras Psiquiatr. 2002;24(S3):7-12.

21. Organização Mundial de Saúde. Classificação de transtornos mentais e de comportamento da CID-10. Descrições clínicas e diretrizes diagnósticas. Porto Alegre: Artes Médicas; 1993.

22. Cordás TA. Transtornos alimentares: classificação e diagnóstico. Rev Psiquiatr Clin. 2004;31(4):154-7.

23. Fairburn CG, Harrison PJ. Eating disorders. Lancet. 2003;361:407-16.

24. Tiggemann M. Media exposure, body dissatisfaction and disordered eating: television and magazines are not the same! Eur Eat Disord Rev. 2003;11(5):41830.

25. Raevuori A, Kaprio J, Hoek HW, Sihvola E, Rissanen A, Keski-Rahkonen A Anorexia and bulimia nervosa in same-sex and opposite-sex twins: lack of association with twin type in a nationwide study of finnish twins. Am J Psychiatry. 2008;165(12):160410.

26. Currin L, Schmidt U, Treasure J, Jick H. Time trends in eating disorder incidence. $\mathrm{Br} \mathrm{J}$ Psychiatr. 2005; 186:132-5.

27. Gadalla T, Piran N. Eating disorders and substance abuse in Canadian men and women: a national study. Eating Disord. 2007b;15:189-203.

28. Krug I, Treasure J, Anderluh M, Bellodi L, Cellini E, di Bernardo M, et al. Present and lifetime comorbidity of tobacco, alcohol and drug use in eating disorders: a European Multicenter Study. Drug Alcohol Depend. 2008;97:169-79.

29. Gadalla T. Psychiatric comorbidity in eating disorders: a comparison of men and women. J Men's Health. 2008a;5(3):209-17.

30. Blinder BJ, Cumella EJ, Sanathara VA. Psychiatric comorbidities of female inpatients with eating disorders. Psychosom Med. 2006;68:454-62.

31. Dansky BS, Brewerton TD, Kilpatrick DG. Comorbidity of bulimianervosa and alcohol use disorders: results from the national women's study. Int J Eat Disord. 2000;27:180-90.

32. Piran N, Gadalla T. Eating disorders and substance abuse in Canadian women: a national study. Addiction. 2006;102:105-13.

33. Vilela JEM, Lamounier JA, Dellaretti Filho MA; Barros Neto JR; Horta GM. Transtornos alimentares 
em escolares. J Pediatr (Rio J.). 2004;80(1):49-54.

34. Souza FGM, Martins MCR, Monteiro FCC, Menezes Neto GC, Ribeiro IB. Anorexia e bulimia nervosa em alunas da Faculdade de Medicina da Universidade Federal do Ceará - UFC. Rev Psiq Clín. 2002;29(4):172-80

35. Alves E, Vasconcelos FAG, Calvo MCM, Neves J. Prevalência de sintomas de anorexia nervosa e insatisfação com a imagem corporal em adolescentes do sexo feminino do Município de Florianópolis, Santa Catarina, Brasil. Cad Saúde Pública. 2008;24(3):503-12.

36. Greenfield, S.F. Women and substance use disorders. In: Jensvold MF, Halbreich U, Hamilton JA, editors. Psychopharmacology and women. Sex, gender, and hormones. Washington, DC: American Psychiatric Press; 1996. p.299-321.

37. Cherpitel CJ, Ye Y. Trends in alcohol- and drugrelated ED and primary care visits: data from three US National Surveys (1995-2005). Am J Drug Alcohol Abuse. 2008;34(5):576-83.

38. Wilsnack RW, Wilsnack SC, editors. Gender and Alcohol: Individual and Social Perspectives. New Brunswick, NJ: Rutgers Center of Alcohol Studies; 1997.

39. Bloomfield K, Gmel G, Neve R, Mustonen H. Investigating gender convergence in alcohol consumption in Finland, Germany, The Netherlands, and Switzerland: a repeated survey analysis. Substance Abuse. 2001;22:39-53.

40. Keyes KM, Martins SS, Hasin DS. Past 12-month and lifetime comorbidity and poly-drug use of ecstasy users among young adults in the United States: Results from the National Epidemiologic Survey on Alcohol and Related Conditions. Drug Alcohol Depend. 2008;97(1-2):139-49.

41. Kerr-Corrêa F, Tucci AM, Hegedus AM, Trinca LA, de Oliveira JB, Floripes TM, Kerr LR. Drinking patterns between men and women in two distinct Brazilian communities. Rev Bras Psiquiatr. 2008;30(3):235-42.
42. Dunne F J, Galatopoulos C, Schipperheijn JM. Gender differences in psychiatric morbidity among alcohol misusers. Compr Psychiatr. 1993;34(2):95101.

43. Schuckit MA, Tipp JE, Bergman M, Reich W, Hesselbrock VM, Smith TL. Comparison of induced and independent major depressive disorders in 2,945 alcoholics. Am J Psychiatr. 1997;154:948-57.

44. Gadalla T, Piran N. Co-occurrence of eating disorders and alcohol use disorders in women: a meta-analysis. Arch Womens Ment Health. 2007a;10:133-40.

45. Franko DL, Dorer DJ, Keel PK, Jackson S, Manzo MP, Herzog DB. How do eating disorders and alcohol use disorder influence each other? Int J Eat Disord. 2005;38(3):200-7.

46. Luce $\mathrm{KH}$, Engler PA, Crowther JH. Eating disorders and alcohol use: group differences in consumption rates and drinking rates. Eat Behav. 2007;8:177-84.

47. Wiederman MW, Pryor T. Multi-impulsivity among women with bulimia nervosa. Int $\mathrm{J}$ Eat Disord. 1996;20(4):359-65.

48. Gadalla TM. Eating disorders and associated psychiatric comorbidity in elderly Canadian women. Arch Womens Ment Health. 2008b;11(5-6):357-62

49. Krahn DD, Kurth CL, Gomberg E, Drewnowski A. Pathological dieting and alcohol use in college women - a continuum of behaviors. Eat Behav. 2005;6:43-52.

50. Benjamin L, Wulfert E. Dispositional correlates of addictive behavior in college women: binge eating and heavy drinking. Eat Behav. 2005;6:197-209.

51. Piran N, Robinson SR. The association between disordered eating and substance use and abuse in women: a community-based investigation. Women Health. 2006;44(1):1-20

52. Keel PK, Dorer DJ, Eddy KT, Franko D. Predictors of mortality in eating disorders. Arch Gen Psychiatr. 2003;60:179-83. 